\title{
A Study on Testicular Development in Apteronotus albifrons
}

\author{
Chengxu $\mathrm{HA}^{1}$, Cuihua YANG ${ }^{1}$, Wei WANG ${ }^{1}$, Yunzhong $\mathrm{WANG}^{1}$ \& Jiguang $\mathrm{Qi}^{1}$ \\ ${ }^{1}$ Qingdao Marine Science and Technology Museum, Qingdao, China \\ Correspondence: Jiguang Qi, Qingdao Marine Science and Technology Museum, Qingdao, China. E-mail: \\ qijiguang@qd.shandong.cn
}

Received: July 27, 2020

Accepted: September 1, 2020

Online Published: September 8, 2020

doi:10.5539/jmbr.v10n1p82

URL: https://doi.org/10.5539/jmbr.v10n1p82

\begin{abstract}
Apteronotus albifrons has its cloacal aperture located in the front of the body and on the back of the lower jaw. A. albifrons possess a pair of testes attached on the mesentery below its liver on the back of the abdominal cavity. In the early days of development, the testes are in translucent elongated thin strips in nude colour. As they develop, the testes will grow thicker and become white or creamy white in color. The rear end of the testes will split whereas the front end will merge to form the vas deferens which is connected to the external environment through the cloacal aperture. Testes of A. albifrons are lobular. There are six stages in its development. At 6 months of age, the testis structure shows clear medium with primary spermatocytes and abundant spermatogonia, suggesting that development is into the stageII. At 8 months of age, testes in male $A$. albifrons are in the shape of a thin rod in white with blood vessels in presence. Germ cells consist of spermatogonia, primary spermatocytes and spermatocytes, indicating stageIII development. At 11 months of age, testes in male A. albifrons grow fuller and are in creamy white with apparent blood vessels present. Primary spermatocytes, secondary spermatocytes, spermatids and a small amount of sperms are president, indicating stageIV of development. At 15 months of age, testes in male A. albifrons swells and consist of a large amount of sperms, reaching full maturity. This is the stageVIof development, after which is the stage featured by testes after spermiation.
\end{abstract}

Keywords: Apteronotus albifrons, Testis, Development

Gonadal development is vital to the reproduction and the existence of fish population. It is also one of the most crucial factors in fish reproduction. Research on testis development in fish species have been the interest of scholars for years. By far, histological and molecular biological methods have been used to look into things such as gonadal differentiation in fish, morphological features of each testes development stage and its time phasing as well as the molecular regulation in testicular development (Kaneko et al., 2015; Fan et al., 2017; Hsu et al., 2018).

Apteronotus albifrons is of Gymnotiformes order and Apteronotidae family (Wan et al., 2002). A. albifrons has a mild temperament and they are amazingly beautiful swimmers which gained popularity among hobbyists. $A$. albifrons has no dorsal fin and is weakly electric. They are very sensitive to water quality and are extremely difficult to breed in captivity. Research on A. albifrons reproduction is very few. Morphological and histological observations in terms of $A$. albifrons testicular development in captive breeding are aimed at understanding the patterns in its testicular development to enrich studies on its biological productivity. It also helps to get a solid grasp on A. albifrons reproduction patterns, thus providing theoretical foundation for artificial breeding of the species, which will boast significant relevance to the protection and development of this species resource.

\section{Material and Methodology}

\subsection{Experimental Fish}

Take 200 A. albifrons and keep in the following condition: water temperature $28 \pm 0.5^{\circ} \mathrm{C}, \mathrm{pH} 7.5 \pm 0.5$, lighting $16 \mathrm{hr}$ : 8hr (lighting: dark). Feed with nematode and bloodworm once daily respectively. Sex will be determined after anatomy. Take 10 fish each at 4 months of age, 5, 6, 7, 8, 9, 10,11, 12, 13, 14 and 15 months of age respectively.

\subsection{Sample Measurement and Morphological Observation of the Gonad}

Measure individual body length, total length, body height, width and weight. Determine the sex via anatomy and observe gonad morphology. Take the gonad and keep it fixed in Bouin solution for 24 hours, then move to be preserved in $70 \%$ ethanol solution. 


\subsection{Tissue Section and Observation}

Use paraffin wax and follow the steps: (1) Dehydration. 70\% ethanol (45 min), 80\% ethanol (45 $\mathrm{min}), 90 \%$ ethanol (45 min), 95\% ethanol (45 min), 100\% ethanol 1 (30 min), 100\% ethanol 2 (30 min). (2) Clearing. 1:1 benzene-alcohol (40min), xylol 1 (40min), xylol 2 (40min). (3) Wax infiltration. 3:1 wax (30 min), 1:1 wax (45min), purified wax $1(30 \mathrm{~min})$, purified wax $2(30 \mathrm{~min})$. (4) Embedding and blocking out. (5) Sectioning. Use KD-3358 microtome to section the tissues. Thickness is $4 \mu \mathrm{m}$. (6) Flattening. Flatten and dry the slice with a temperature of $70^{\circ} \mathrm{C}$. (7) HE staining. Conduct HE stain $12 \mathrm{hrs}$ after the section is flattened. (8) Mounting with gum. Check the slice under microscope and preserve accordingly.

\subsection{Data Analysis and Processing}

Measure the biological parameters of the experimental fish, including body weight (BW), total length (TL), body length (BL) and gonadal weight (GW) etc. Calculate relevant gonadal development indices: Gonadosomatic index $(\mathrm{GSI})=\mathrm{GW} / \mathrm{BW} \times \mathrm{x}$ 100. Analyze the data with Microsoft Excel 2010. Mean $\pm \mathrm{SD}$ is used for both measuring the data and calculating GSI. Conduct ANOVA analysis (SPSS software, V19.0) with the data above and check significance of difference. Note significance of difference $(P)$ as 0.05 . If $P \leq 0.05$ then there is statistical difference, if not, then no statistical difference.

\section{Results and Analysis}

\subsection{Morphology and Structure of the Testes}

A. albifrons has its cloacal aperture located in the front of the body and on the back of the lower jaw. A. albifrons possesses a pair of testes attached on the mesentery below its liver on the back of the abdominal cavity. In the early days of development, the testes are in translucent elongated strips in nude colour. As they develop, the testes will grow thicker and become white or creamy white in color. The rear end of the testes will split whereas the front end will merge to form the vas deferens which is connected to the external environment through the cloacal aperture.

\subsection{Testicular Development Stages and Histological Observation}

Referring to the testicular development staging methods by Yun Liu (Liu, 1933), Yaoguang Zhang (Zhang, Luo, $\&$ Zhong, 1992) and Yundong Lou (Lou, 1980) et al. and combined with the morphological features of $A$. albifrons testes and its germ cell distribution, six stages are identified during the development course of the testes:

At stageI, testes are in pairs and closely attached to the two sides of the abdominal cavity. They are in transparent and elongated thin strips which are hard to distinguish. Through histological studies, spermatogonia are randomly present and scattered among the connective tissues.

At stageII, testes are in translucent and elongated thin strips with nude color. Blood vessels are inapparent. However, testes and ovaries can be distinguished at this stage with naked eyes. Early seminiferous lobules can be visible. Primary spermatocytes are present with the number of spermatogonia increasing and apparent medium among tissues.

At stageIII, testes are in the shape of a thin rod in white with blood vessels present. Seminiferous lobule becomes larger. At this stage, germ cells in the testes generally consist of spermatogonia, primary spermatocytes and secondary spermatocytes. Primary spermatocytes are round or oval shaped. They have a smaller diameter than the spermatogonia with rich chromosomes in the nuclei and a violet colour pigmentation. The nucleus is going through the first meiosis. Secondary spermatocytes are smaller than primary spermatocytes with its nucleus in violet colour and basophilia continuing to enhance. Compared to the second stage, primary spermatocytes count is significantly increased.

At stageIV sees further development of the testes. They become larger, fuller and creamy white with clear distribution of the blood vessels. Seminiferous lobules continue to grow and so is the seminiferous lobule cavity. Primary spermatocytes, secondary spermatocytes, spermatids and a small amount of sperms along with others of the same cell types are piled up. Secondary spermatocytes, after the second mitosis, develop into spermatids that are irregular in shape with no apparent cytoplasm. Only the round nucleus is visible which has high basophilia and a violet colour. Sperm counts in mid and later stages gradually go up.

At stageV, testes are in creamy white blocks and reach maximum sizes. Blood vessels further develop, and the distribution is more distinctive. Testes are fully grown and reach full maturity. In this stage, the majority of germ cells in the testes are comprised of androcytes and sperms in metamorphosis. Sperms in great density swirled in and fulfilled the seminiferous lobule cavity, showing a deep blue colour. The sperm is the smallest cell in the 
testes. It is a metamorphotic structure after the spermatid matures. The nuclei are clustered at the head of the sperm. Cells are in dark colours, in deep violet. A small number of spermatids and primary spermatocytes are visible on the edge of the seminiferous lobules. Male A. albifrons can discharge seminal fluid at this stage for multiple times. Emptied seminiferous lobule cavity can be visible.

At stageVI, testes become degenerated or have finished spermiation. Its size will decrease and the colour becomes light pink. Sperms in most seminiferous lobule cavities are discharged with a small amount of "old" sperms or undischarged sperms remaining to be absorbed. The rest are a very rare amount of spermatogonia and spermatocytes.

\subsection{Male Gonadal Development Process}
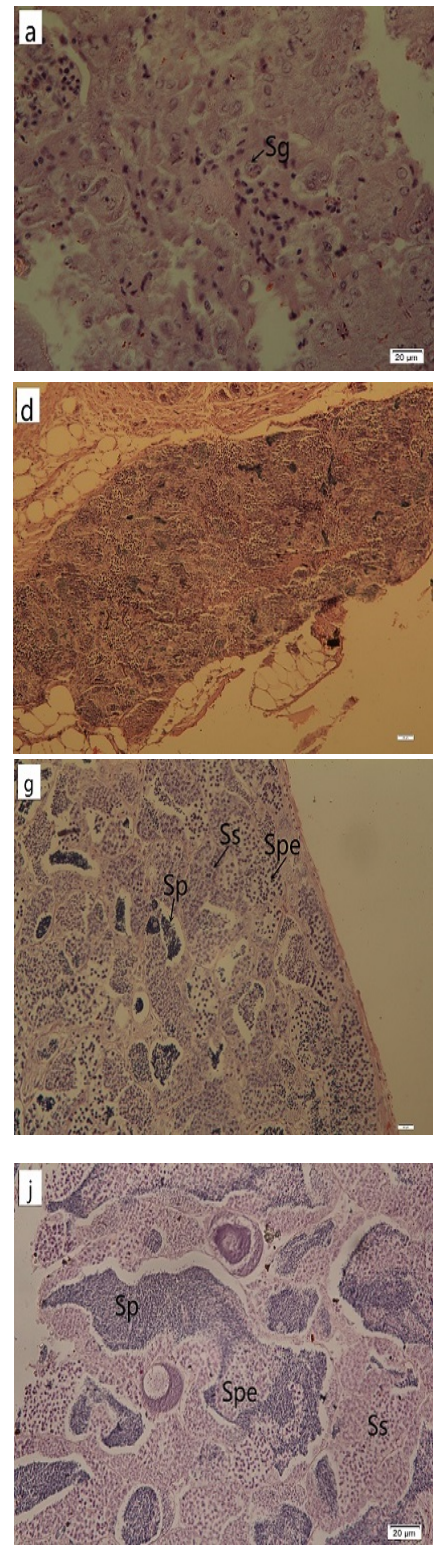
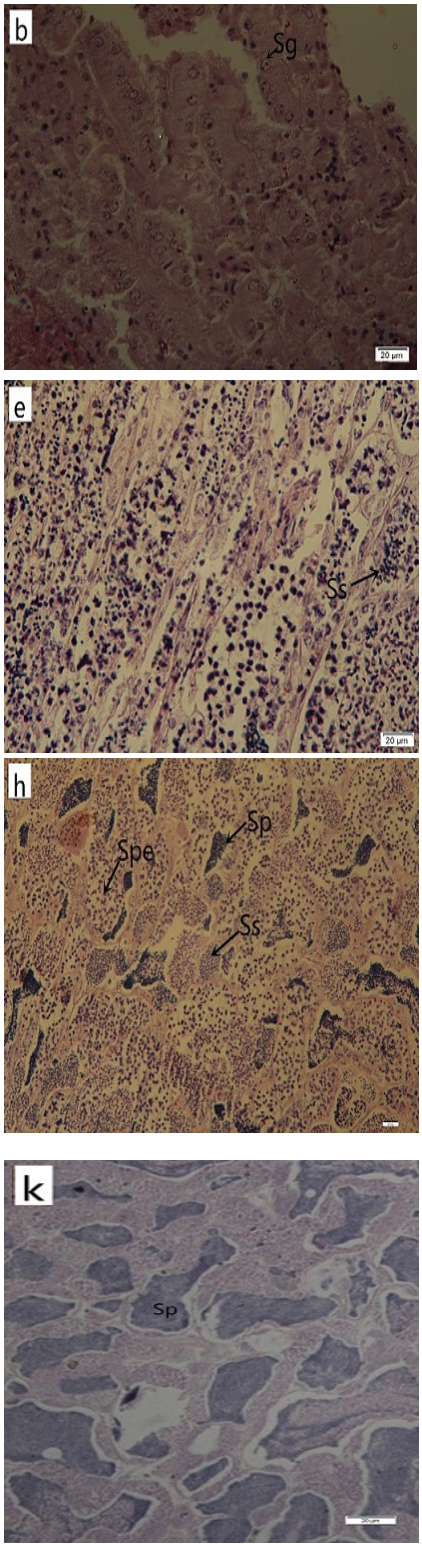
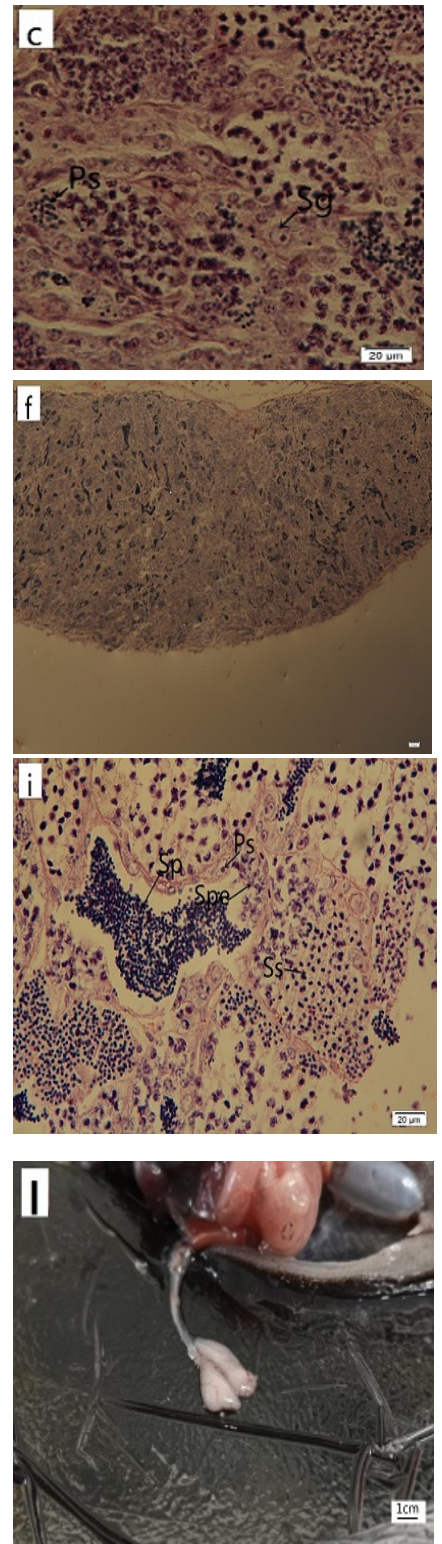

Figure 1. Testicular development of Apteronotus albifrons

a. stageI, showing testis of 4-month-old male fish; b. stageI, showing testis of 5-month-old male fish; c: stageII, showing testis of 6-month-old male fish; d: stageII,showing testis of 8-month-old male fish; e: stageIII, showing testis of 9-month-old male fish; f: stageIV, showing testis of 11-month-old male fish; g: stageIV, showing testis of 11-month-old male fish; h: g: stageIV, showing testis of 12-month-old male fish; i: stageIV, showing testis of 12-month-old male fish; $\mathrm{j}$ : stageIV,showing testis of 14-month-old male fish; $\mathrm{k}$ : stageV, showing testis of 15-month-old male fish; 1: mature testis of 15-month-old male fish; Sg: spermatogonia; Ps: Primary spermatocyte; Ss: Secondary spermatocyte; Sp: spermatids; Sp: Sperms. 
The correlation among male A. albifrons development parameters, its gonadal development and month of age can be seen from Table 1. At 6,7months of age, male A. albifrons is in testes development StageII; at $8,9,10$ months of age in StageIII at 11 to 14 months of age in Stage IV, and at $15^{\text {th }}$ months of age in StageV. After spermiation, testes development then moves to StageVI.

Changes in male A. albifrons GSIs can be seen from Figure 2. As the gonad starts to develop, testes increase in weight and size and parent GSI starts to climb. Development accelerates in the StageIII and peaks during the StageV at $0.612 \pm 0.06$ which is significantly higher than the $0.16 \pm 0.03$ identified during the StageIII in ovaries.

Table 1. Correlation between Testicular Development and Months of age in Apteronotus Albifrons

\begin{tabular}{lllllll}
\hline Months of age & Sample number & Overall length/mm & Body length/mm & Body height/mm & Body weight/g & Stage \\
\hline 4 & 10 & $66.3 \pm 1.8$ & $52.6 \pm 1.8$ & $10.4 \pm 0.5$ & $0.80 \pm 0.08$ & I \\
5 & 10 & $74.6 \pm 3.8$ & $64.3 \pm 2.1$ & $14.7 \pm 0.5$ & $1.48 \pm 0.06$ & I \\
6 & 10 & $82.9 \pm 2.4$ & $72.5 \pm 0.2$ & $17.9 \pm 0.8$ & $2.19 \pm 0.15$ & II \\
7 & 10 & $89.9 \pm 2.0$ & $79.6 \pm 2.8$ & $19.6 \pm 1.4$ & $3.69 \pm 0.05$ & II \\
8 & 10 & $103.6 \pm 2.5$ & $86.6 \pm 1.8$ & $20.6 \pm 1.3$ & $5.39 \pm 0.57$ & III \\
9 & 10 & $126.4 \pm 5.3$ & $102.2 \pm 4.7$ & $25.4 \pm 1.3$ & $7.98 \pm 0.53$ & III \\
10 & 10 & $156.3 \pm 6.2$ & $139.9 \pm 6.4$ & $34.6 \pm 1.3$ & $15.49 \pm 0.76$ & III \\
11 & 10 & $198.3 \pm 18.3$ & $175.7 \pm 16.2$ & $42.7 \pm 6.9$ & $20.99 \pm 3.24$ & IV \\
12 & 10 & $268.3 \pm 15.7$ & $240.0 \pm 12.1$ & $53.6 \pm 7.3$ & $54.28 \pm 3.18$ & IV \\
15 & 10 & $323.7 \pm 19.5$ & $282.0 \pm 18.1$ & $66.6 \pm 8.9$ & $69.26 \pm 5.27$ & V \\
\hline
\end{tabular}

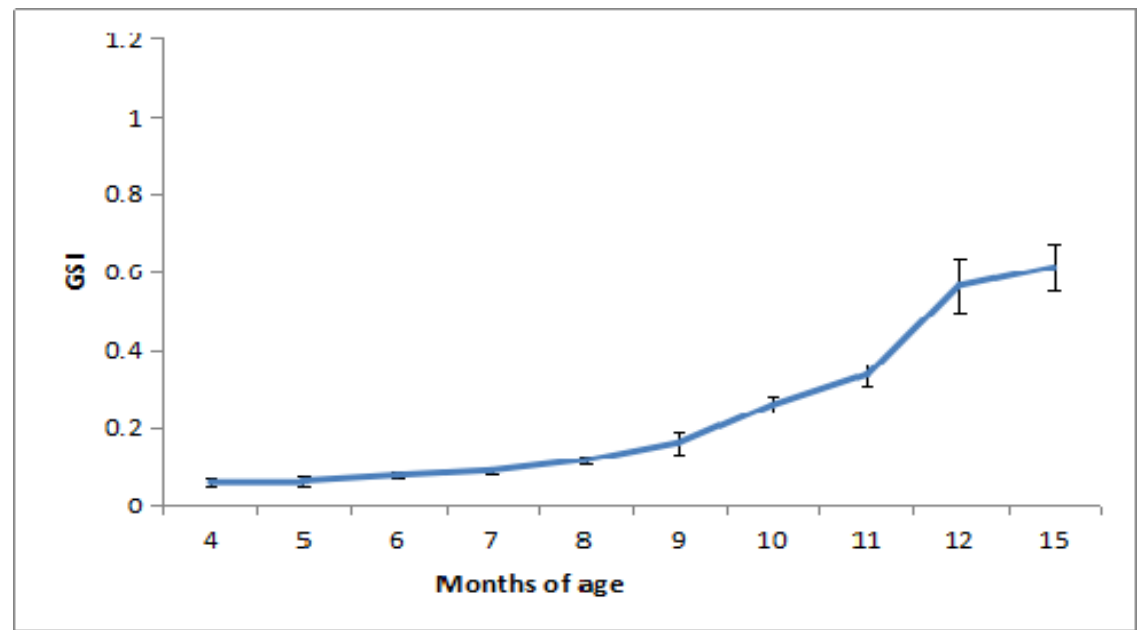

Figure 2. Monthly variations in GSI of male

\section{Discussion}

\subsection{Male A. albifrons Gonadal Development Features}

Studies on fish testicular development are far less detailed than those on ovarian development. There is yet to be a standard set of demarcations on the staging of testicular development. Based on testicular anatomy and histological features, some researchers have divided testicular development into the following five stages, i.e. immature, developing, maturing, spawning and spent (Akayli et al., 2013). Chinese researchers normally divide the process into 6 stages. As spermatogonia gradually mature, they go through five major stages, i.e. spermatogonia, primary spermatocyte, secondary spermatocyte, spermatid and sperm. A. albifrons testicular development demonstrates a "catch-up growth" phenomenon that goes from synchronized development to non-sync development and then to synchronized development again. This phenomenon is also present in the testicular development in teleost such as Eleutheronema tetradactylum (Lan et al., n. d.) and Anguilla japonica (D. Lin \& H. R. Lin, 1984). The key features are that during the At stageIand stageII, spermatogonia and spermatocytes proliferate, and development is basically in sync. By the stageIII, the seminal vesicle near the seminiferous lobule cavity will first mature whereas the seminal vesicle near the intralobular interstitium 
develops slowly which demonstrates the non-sync phenomenon. By the stageIV, seminiferous lobule cavity starts to see mature sperms and all sperms will gradually mature and continue to be released into the seminiferous lobule cavity. When developed to the stageV, the seminiferous lobule cavity, efferent tube, and vas deferens are filled with sperms. Development is then again in sync (Lan et al., n. d.).

Based on the histological structure and germ cell arrangement as well as development features, two main types of testicular structure are distinguished: a tubular and a lobular (Nagaham, 1983; Billard, 1986). There is no seminiferous lobule in tubular types which are not commonly seen in teleost fish species. Based on the arrangement of the seminiferous lobules, there are two subtypes of lobular testicular type, namely, radial type and ampulla type (Lan et al., n. d.). With radial type, the seminiferous lobules are specifically arranged in a radial layout in the testes, examples of which could be Leiocassis crassilabris (Chen et al., 2008) and Eleutheronema tetradactylum (Lan et al., n. d.), whereas with ampulla type, the arrangement is usually irregular as seen in spinibarbus caldwelli (Lin, You, \& Su, 2003). Research shows that $A$. albifrons testes are made up of many seminiferous lobules in which there are seminal vesicles where spermatids develop to maturity and then are released to seminiferous lobule cavity. Eventually, matured sperms are stored in the seminiferous lobule cavity and vas deferens. Seminiferous lobules are randomly arranged in A. albifrons testes. Its testicular structure falls under the ampulla type.

\subsection{A. albifrons GSI and Reproduction}

Compared to most teleost, male A. albifrons has relatively low GSI levels. Sexually matured male Xenocypris davidi (Bian \& Yang, 1992) has a GSI as high as 7.8 whereas male Schizopygopsismalacanthus baoxingensis GSI can reach 5.41 during the peak of the breeding season. GSI of male Oreochromis niloticus ranges between 1.28 to 1.49 . In contrast, male A. albifrons GSI is only 0.5 at the maximum. This has made it more difficult for the fish to be artificially bred. As the gonad is too small compared to the size and weight of the body, it is extremely difficult to obtain matured sperms externally (Xu et al., 2015). Gonadal development is regulated by individual hormone levels. Environmental factors also play a role in the reproduction of fish (Xu et al., 2017). Many environmental elements, such as meteorological and hydrological factors (water temperature, lighting), water physical and chemical factors (salinity, $\mathrm{pH}$ ), fish feed and spawning substrates etc. can all impact fish reproduction (Wen \& Lin, 2001; Song \& Wen, 2005). With artificial breeding, feed and water quality are usually good. Gonads develop very fast in apteronotus albifrons, however, non-sync development between male and female A. albifrons were identified during the research which increased the difficulty for artificial breeding.

\section{Conflict of interests}

The authors declare that there is no conflict of interests regarding the publication of this paper.

\section{References}

Akayli et al. (2013). Testes development and maturity classification of albacore (Thunnus alalunga (Bonaterre, 1788)) from the Eastern Mediterranean Sea. Journal of Applied Ichthyology, 29(4), 901-905.

Bian, W., \& Yang, A. S. (1992). Study on the gonadal development in xenocypris davidi bleeker. Acta Hydrobiologica Sinica, 16(4), 346-355.

Billard, R. (1986). Sperm atogenesis and sperm atology of some teleostfish species. ReproductionNutrition Développement, 26(4), 877-920.

Chen et al. (2008). Study on the microstructures of testis and spermatogenesis of Leiocassis crassilabris. Fresh water Fisheries, 38(1), 6-10.

Fan et al. (2017). Significant association of cyp19a promoter methylation with environmental factors and gonadal differentiation in olive flounder Paralichthys olivaceus. Comparative Biochemistry and Physiology Part A: Molecular \& Integrative Physiology, 208, 70-79.

Hsu et al. (2018). Changes in the morphology and gene expression of developing zebrafish gonads. General and Comparative Endocrinology, 265, 154-159.

Kaneko et al. (2015). Gonadal soma-derived factor (gsdf), a TGF-beta superfamily gene, induces testis differentiation in the teleost fish Oreochromis niloticus. Molecular \& Cellular Endocrinology, 415, 87-99.

Lan et al. (n. d.). Histological and ultrastructure of testis development and spermatogenesis in Fourfinger threadfin, Eleutheronema tetradactylum (Show, 1804). Journal of Fishery Sciences of China. Retrieved from http://kns.cnki.net/kcms/detail/11.3446.S.20191223.1516.003.html 
Lin, D. J., You, Y. L., \& Su, M. (2003). Studies on testicular histology and spermatogenesis of teleost,Spinibarbus caldwelli(Nichols). Acta Hydrobiologica Sinica, 27(6), 563-571.

Lin, D., \& Lin, H. R. (1984). Studies on the breeding biology of the eel(Anguilla japonica Temminck \&Schlegel) 3. Histological and cytological studies on the gonadal development of eel. ActaHydrobiologica Sinica, 8(2), 157-170.

Liu, J., Liu, G. A., \& Cheng, S. Q. (1983). Studies on the gonadal development of Tilapia nilotica[J]. Acta Hydrobiologca Sinca, 8(1), 17-32.

Liu, Y. (1933). Reproductive Physiology of Chinese Cultured Fishes. Beijing: China Agriculture Press, 22-30.

Lou, Y. D. (1980). Histology and embryology. Beijing: China Agriculture Press, 131-137.

Nagaham, A. Y. (1983). The functionalm orphology ofteleostgonads in W S H oar,D JRandall,E N Donaldson, eds. Fish Physiology, 9(6), 223-275. PartA. Endocrine tissue and horm one. New York: Academ icPress.

Song, H. X., \& Wen, H. S. (2005). Histological studies on the ovaian development and its mechanism of regulation-controlling in cultured Japanese flounder Paralichthys olivaceus. Transactions of Oceanology and Limnology, 4, 75-83.

Wan et al. (2002). Freshwater ornamental fish. Science Press, 76-77.

Wen, H. S., \& Lin, H. R. (2001). Effect of environmental factors on gonadal maturation as well as its ovulation and spawning in teleosts. Chinese Journal Of Applied Ecology, 12(1), 151-155.

Xu et al. (2015). Gonadal development of artificially-cultured discus fish (Symphysodon spp.). Journal of Anhui Agricultural University, 42(1), 115-123.

$\mathrm{Xu}$ et al. (2017). Journal of Yangtze University (Natural Science Edition), 14(6), 43-48.

Zhang, Y. G., Luo, Q. S., \& Zhong, M. C. (1992). Studies on the developmental stages of testis,spermatogenesis and spermatoleosis in Leiocassis longirostris. Zoological Research, 13(3), 281-287.

Zhou, C. P. (2007). Reproduce biology of Schizopygopsis malacanthus baoxingensis. Sichuan Agricultural University.

\section{Copyrights}

Copyright for this article is retained by the author(s), with first publication rights granted to the journal.

This is an open-access article distributed under the terms and conditions of the Creative Commons Attribution license (http://creativecommons.org/licenses/by/4.0/). 